\title{
The Future of Food Colloids: Next-Generation Nanoparticle Delivery Systems
}

\author{
David Julian McClements \\ Department of Food Science, University of Massachusetts Amherst, Amherst, MA 01003, \\ USA
}

mcclements@foodsci.umass.edu; 4135452275

Journal: Current Opinion in Colloid Science

Submitted: June, 2016

\begin{abstract}
The area of food colloids is rapidly evolving. Food researchers are continuing to use theoretical, modeling, and experimental approaches to understand colloidal phenomena in foods and in the gastrointestinal tract, as well as to use colloid science to design foods with novel or improved functional attributes. In this article, a brief survey of potential topics of future study in food colloids is given, including the application of colloid science in improving food security, enhancing human health, and ensuring food quality and safety. Special emphasis is given to the development of next-generation delivery systems, such as mixed nanoparticle systems, nanoclusters, Trojan-horse nanoparticles, and environmentally responsive nanoparticles.
\end{abstract}

Keywords: nanoparticles; nanoemulsions; Trojan-horse nanoparticles; delivery systems; gastrointestinal tract

\section{Introduction}

It is difficult to accurately predict the future of any scientific discipline. If one looked at the field of food colloids when I started working in this area in the late 1980's it would have been difficult to predict the research areas that currently dominate the field, such as understanding the colloidal basis of food digestion and the development of

(C) 2016. This manuscript version is made available under the Elsevier user license http://www.elsevier.com/open-access/userlicense/1.0/ 
structured delivery systems to control the gastrointestinal fate of nutrients and nutraceuticals. In those days, a much greater emphasis was given to understanding the various kinds of colloidal and interfacial phenomena that occurred within foods, and in relating colloidal phenomena to the bulk physicochemical properties of foods, particularly stability and rheology. The discipline has evolved considerably since then, with a much stronger emphasis on utilizing colloidal principles to rationally design new structures and functionalities in foods, and with a greater interest in understanding the colloidal phenomena occurring within the human body after a food has been ingested. In some respects, the future of food colloids will look similar to the past and present. Many foods are highly complex materials that contain biopolymers and colloidal particles, and so it will continue to be important to use colloidal theories and techniques to understand, predict, and manipulate their behavior. Thus, many current areas of research will simply broaden and deepen as a wider range of materials is studied using a greater number of analytical approaches.

In other respects, the future of food colloids may look very different from the current discipline. It is likely that new topics of study will be identified and pursued, and that the introduction of novel analytical and computational tools will open up new areas of knowledge to be explored. Indeed, the problems that can be studied in a particular scientific discipline are often limited by the availability of suitable analytical tools to provide information about them.

In this article, I will first outline potential new areas of research in food colloids that are either driven by important societal problems that need to be addressed or by the introduction of new research tools. I will then focus on a particular area that is likely to rapidly expand in the near future: next-generation nanoparticle delivery systems.

\section{Origin of New Research Areas: Problem Driven}

New topics of research are likely to come from a number of directions. A great deal of science and technology is motivated by practical problems that need to be solved in the real world. Much of current research in colloid science is focused on the gastrointestinal fate of foods because of the greater appreciation of the close link between human diet and health, and the possibility of manipulating food composition and 
structure to improve human health and wellbeing through dietary interventions [1, 2]. Overconsumption of certain types of food components is leading to a variety of chronic diseases that adversely affect human well-being and that cost society considerable amounts of money in lost productivity and increased health care costs, e.g., heart disease, obesity, diabetes, hypertension, and cancer [3-6]. Conversely, fortification of foods with other types of food components (nutraceuticals) may be able to inhibit or prevent chronic diseases [7-9]. Consequently, a great deal of current research in food colloids is focusing on using colloid and interface science to structure foods so as to improve their nutritional profiles and alter their gastrointestinal fate [1, 10-13]. In the future, colloid science may also be useful for tackling other existing or emerging societal challenges. A number of potential areas of focus are outlined in this section.

\subsection{Food processing and sustainability}

The continued growth of the human population means that an increasing amount of food will be required to provide a nutritious, healthy, and sustainable diet for all [14, 15]. Humans will need to generate more food, and to utilize and distribute it more wisely. The principles and tools of colloid science may play an important role in developing a more efficient and sustainable food supply. Colloid science may improve the processes used to isolate, purify, and process foods and food ingredients so as to make them more efficient and sustainable. For example, waste streams from food processing plants may be converted into valuable ingredients that can be used for food or non-food applications, such as emulsifiers, thickening agents, gelling agents, foaming agents, or nutraceuticals $[16,17]$. Colloid science may also be useful for optimizing the efficiency of various processing operations, such as filtration, centrifugation, and thermal processing [18]. For example, an understanding of the attractive and repulsive interactions between colloidal particles can be used to promote or prevent their aggregation, which may help to separate them or keep them in suspension. Similarly, an understanding of the factors that impact the coalescence of oil droplets can be utilized to improve the separation of bioactive lipids (such as $\omega-3$ oils) from natural and sustainable sources, such as the oil bodies in algae and plant seeds. Colloid science may also be useful for developing delivery systems that can increase the effectiveness and reduce the environmental impact of antimicrobials, pesticides, herbicides, etc. For example, nanoemulsion-based delivery 
systems are being developed to improve the efficacy of natural antimicrobial agents, such as essential oils [19-23].

\subsection{Diet and health}

Until fairly recently the majority of the research in food colloids was focused on understanding and controlling the processes that occur within foods themselves. For instance, a great deal of research was carried out on understanding the factors that influence the stability or rheology of oil-in-water emulsions. Recently, there has been increasing interest in understanding the colloidal and interfacial processes that occur within the gastrointestinal tract (GIT) after the food is ingested [1, 2, 12, 24-26]. For example, the influence of the composition and structure of emulsions on their gastrointestinal fate has been an area that has been the focus of a large research effort in recent years $[2,10]$. In the future, food scientists may also be interested in controlling the colloidal and interfacial processes that occur within the systemic circulation and specific tissues after a digested food has been absorbed by the human body. This knowledge may lead to new approaches for fighting cancer, coronary heart disease, or other chronic diseases [27]. For example, a food emulsion may be designed on the nanoscale to breakdown in the GIT and form specific nanostructures (mixed micelles and vesicles) that promote the absorption of beneficial nutraceuticals or nutrients. At the same time, the initial food emulsion could also be designed so that it is processed within the epithelium cells so as to form chylomicrons with specific dimensions and compositions. After being released into the systemic circulation these natural nanoparticles may be preferentially adsorbed by tumors, thereby enabling the delivery of anticancer nutraceutical components to the site of action more effectively. Thus, a highly integrated colloidal approach could be used to design the behavior of foods from before, during, and after ingestion [27].

There has been a lot of interest in utilizing both organic and inorganic nanoparticles in foods to improve their safety, quality, or nutritional value [28, 29]. However, the incorporation of these nanoparticles may alter the gastrointestinal fate and therefore toxicity of foods. Consequently, it is important to understand how both organic and inorganic food-grade nanoparticles behave in the human body [10, 30]. Nanoparticles (1 to $100 \mathrm{~nm}$ ) are really a special class of colloidal particles (1 to $1000 \mathrm{~nm}$ ) and therefore 
the principles and techniques of colloid science will prove especially important for understanding their biological fate.

The human microbiome is known to play a major role in determining human health and wellbeing, and can be manipulated through diet [31, 32]. As knowledge about the specific components within the human diet that lead to a healthy microbiome advance, it may be possible to use colloid science to design foods that deliver an optimized balance of nutrients to the gut microbiota. Alternatively, it may be possible to use food-grade colloidal particles as diagnostic tools to provide information about the status of the human microbiome. For example, a food could contain colloidal particles that produce measurable signals when they encounter certain conditions in the human colon associated with disease states, e.g., specific metabolites produced by gut bacteria. For instance, the colloidal particles may cause a change in the color of the feces, which informs a person to go for a more detailed health check.

\subsection{Food diagnostics}

There have been rapid advances in the development of sensors to monitor the safety and quality of food products, and then report the information obtained to food manufacturers or consumers, e.g., smart labels, microfluidic chips, paper assays, or inproduct probes [33-39]. These sensors may report if a product has received temperature abuse, if it has been contaminated with spoilage or pathogenic microorganisms, if it has reached the end of its shelf life, or if it contains undesirable levels of toxins, pesticides, herbicides, or hormones. Colloid science may play an important role in the development of many of these sensors. For example, colloidal particles could contain sensors that generate a measureable change when they are exposed to specific temperature, light, $\mathrm{pH}$, enzyme, or chemical environments, such as a color change. These sensors could be contained within the interior of the colloidal particle or they may be adsorbed to the particle surface. Alternatively, the colloidal particles may simply aggregate and become turbid when a specific change in environment occurs. Colloidal sensors could be part of food packaging or they could be added into the food itself (provided they are known to be safe and do not effect food quality). 


\subsection{Natural foods}

There is increasing interest by many consumers in consuming natural or minimally processed foods because they are perceived to be healthier and more environmentally friendly [40]. Colloid science may prove to be useful for understanding how these foods behave during processing, transport, utilization, and consumption, which may lead to products with better quality attributes or nutritional properties. In particular, there is great interest in replacing synthetic food ingredients (such as colorants, flavorings, preservatives, emulsifiers, foaming agents, thickeners, and gelling agents) with more natural alternatives, e.g., natural pigments, oils, proteins, polysaccharides, or phospholipids. For instance, there has been a push towards identifying, isolating, and characterizing natural emulsifiers to replace synthetic ones [41-45]. Another area of interest has been in the identification of specific colloidal structures within natural materials (such as fruits and vegetables) that may be utilized as natural delivery systems for bioactive agents. For example, oil bodies are designed by nature to store lipids and protect them from environmental stresses (until they are needed by the plant, animal, or microbe as an energy source to grow), and so it may be useful to design processing operations that leave these structures intact so that they can be utilized as food ingredients [46-50]. Oil bodies consist of lipid droplets containing hydrophobic bioactives and triglycerides that are naturally coated by a layer of phospholipids and proteins. Ideally, sustainable processes could be used to isolate the oil bodies from their natural origin and maintain their structure. Other kinds of colloidal structures in natural foods may also be identified and used for a similar purpose e.g., viruses, bacteria, and yeasts [51].

\subsection{Food safety}

The modern food supply is provided through a highly complex and integrated process with many different stages involved in food production, storage, and transport. It is extremely important to maintain the safety of foods throughout this supply chain. The increasing interest from consumers in having more natural foods, such as organic fruits and vegetables, is causing potential problems with microbial contamination. One area where colloid science may play an important role is in the development of natural antimicrobial delivery systems. For example, nanoemulsion-based delivery systems have been developed to inhibit or kill both pathogenic and spoilage organisms [21-23, 52-55]. 
These systems must be carefully designed to ensure that they are safe, stable, and efficacious for specific applications. Future work may focus on the optimization of these systems, and in identifying their mechanisms of operation.

\section{Origin of New Research Areas: Technology Driven}

A scientific discipline may also move into new research areas due to the introduction of innovative new ideas or techniques from other areas (such as biology, chemistry, physics, or engineering) or from within the discipline itself. In this section, a brief overview of some potential areas for future research based on the introduction of new or emerging technologies is given.

\subsection{Analytical techniques}

The introduction of new analytical tools may revolutionize a particular research discipline. A number of years ago, the widespread commercial availability of light scattering instruments that could rapidly and accurately measure particle size and charge revolutionized the area of food colloids. Researchers could then systematically examine the factors that impacted the formation and stability of colloidal systems and develop structure-function relationships that enabled better understanding, prediction, and control of food properties [56]. It is likely that the utilization of new powerful analytical tools will have a similar transformative effect in the future. An area that is likely to be particularly important in the future is chemical spectroscopy, i.e., the ability to measure the chemical composition of food systems at the nanoscale. This will enable one to monitor the location of different components within a colloidal system and to follow chemical changes as they occur, such as oxidation, reduction, hydrolysis etc.

Understanding and controlling the chemical breakdown of food components is becoming increasingly important for a number of reasons. Food manufacturers are trying to fortify functional foods and beverages with nutrients and nutraceuticals that have potential health benefits, but that are highly susceptible to chemical degradation, such as $\omega-3$ oils, carotenoids, and curcumin [27]. In addition, food manufacturers are also trying to replace synthetic functional ingredients (such as antioxidants, colors, and flavors) with more natural ones, which are often less chemically stable [57, 58]. Improved analytical tools to monitor these chemical changes at the nanoscale would lead to the identification 
of more effective strategies to control them. At present there are a number of chemical microscopy methods that can provide information on the micrometer-scale, such as infrared, Raman, and fluorescent microscopy [59, 60]. However, there are still few methods for providing information on the nanometer-scale. Energy dispersive X-ray spectroscopy (EDS) electron microscopy methods can be used to provide high resolution information about the spatial distribution of certain components in foods and other biological materials [61]. Photoacoustic microscopy can be used to provide information about the composition of concentrated systems on the nanometer scale without dilution [62]. Recently, various scanning probe microscopy methods have been developed that enable chemical composition to be determined on a nanoscale resolution [63]. The further development and application of these methods to food colloids could lead to major advances in understanding and controlling the properties of complex foods.

\subsection{Computer simulation and design}

Computer simulations have been used for many years to model the properties of biopolymer and colloidal systems relevant to foods [64]. These techniques provide valuable insights into the molecular and colloidal properties that impact the behavior of complex systems. One of the main reasons that computer simulation methods have not been utilized more widely by colloid scientists in the food industry is the lack of inexpensive, easy to learn, and simple to use software. The ready availability of commercial software for this purpose would help food scientists to understand and design food colloidal systems more systematically.

In principle, it should be possible to design a colloidal food product with specific properties (optical, rheological, stability, sensory, nutritional) on a computer. This requires knowledge of structure-function relationships that link molecular and colloidal properties to bulk physicochemical and physiological properties. At present, many of the theoretical models and empirical data required to build these relationships is already available [56], although no one has yet attempted to put them all together into a single model. For example, theories are available to relate the size and concentration of the droplets in emulsions to their optical properties [65], to their rheological properties [66], and to their stability [56]. The construction of a single computational model to predict to properties of emulsions, and the experimental determination of how accurately this model 
can actually predicts food emulsion properties, may help to identify new areas of research.

\subsection{3-D Printing}

There has been much interest in assembling foods from the bottom-up using 3-D printing approaches [67]. In principle, a consumer could then download the instructions for making a particular food ("the recipe”), and the 3-D printer would assemble it. The operation of such devices relies on understanding how food components (such as biopolymers and colloids) behave and interact with each other under different conditions [68]. Thus food colloids may play an important role in creating the next generation of 3D printers.

\section{Next-Generation Nanoparticle Delivery Systems}

There has been considerable progress in the development of nanoparticle-based delivery systems for utilization in the food industry [69-73]. Nanoparticles have been fabricated from various types of food-grade ingredients, including lipids, phospholipids, surfactants, proteins, and polysaccharides. Often these ingredients are used in combination to form composite nanoparticles, such as nanoemulsions (lipid core with a protein or surfactant shell), filled microgels (lipid droplets embedded in biopolymer microspheres), complex coacervates (electrostatically linked proteins and polysaccharides), and Maillard complexes (covalently linked proteins and carbohydrates). Food-grade nanoparticles have been shown to be capable of encapsulating, protecting, and releasing bioactive food components, thereby improving their utilization, stability, and effectiveness [74]. Most previous studies have developed a single type of nanoparticle with a relatively simple structure (usually spherical), e.g., lipid [72] , protein $[73,75]$, or phospholipid [76] nanoparticles. In this section, recent work on the development of “next-generation” nanoparticle-based delivery systems is covered.

\subsection{Mixed nanoparticle delivery systems}

As just mentioned, most of the previous work on nanoparticle-based delivery systems in the food industry has focused on the development of a single type of nanoparticle to encapsulate a bioactive agent [73-75]. However, for some applications it 
may be more advantageous to develop mixed nanoparticle delivery systems that contain two or more different kinds of nanoparticles with different functional attributes. For example, it was recently proposed that there might be advantages to encapsulating chemically labile hydrophobic bioactive components within protein nanoparticles that are then mixed with lipid nanoparticles (Figure 1). The protein nanoparticles are designed to protect the encapsulated bioactives from chemical degradation, whereas the lipid nanoparticles are designed to provide a source of digestible triglycerides (TGs) that increase the solubility of the bioactivity in the gastrointestinal fluids. The TGs are rapidly converted to free fatty acids (FFAs) and monoglycerides (MGs) during lipid digestion in the small intestine. The lipid digestion products (FFAs and MGs) then associate with phospholipids and bile salts to form mixed micelles that solubilize the hydrophobic bioactives after they are released from the protein nanoparticles. Recent studies using this concept, have fabricated either curcumin-loaded or tangertin-loaded zein nanoparticles using antisolvent precipitation, and then mixed them with lipid nanoparticles fabricated using microfluidization [77, 78]. In both studies, the bioaccessibility of the hydrophobic bioactive increased as the concentration of lipid nanoparticles was increased due to the resulting increase in the solubilization capacity of the mixed micelle phase.

In principle, many other kinds of mixed delivery system could be developed based on this principle depending on the challenges that have to be overcome for the particular bioactive component utilized. For example, we have recently used mixtures of encapsulated and non-encapsulated lipid nanoparticles to modulate lipid digestion profiles, where the encapsulated particles were trapped in hydrogel beads.

\subsection{Nanoparticle clustering}

The small size of nanoparticles means that they can be used to form threedimensional space-filling particle networks at relatively low nanoparticle concentrations. For example, it has been shown that the heteroaggregation of oppositely charged nanoparticles can be used to form highly viscous solutions or gels [79-81]. These clusters are formed by mixing a suspension of positively charged nanoparticles with a suspension of negatively charged nanoparticles at an appropriate ratio (Figure 2). Nanoparticles with specific charge characteristics can be formed by coating them with 
emulsifiers with different electrical properties. For example, positively charged lipid nanoparticles are formed using cationic emulsifiers (such as proteins below the isoelectric point), whereas negatively charged lipid nanoparticles are formed using anionic emulsifiers (such as proteins above the isoelectric point or anionic surfactants) [79, 81]. This principle may be utilized to form reduced calorie food products with improved or novel textural characteristics. However, the strength of the attractive interaction between the oppositely charged nanoparticles is highly sensitive to $\mathrm{pH}$ and ionic strength, and so these factors would have to be carefully controlled. In principle, any kinds of oppositely charged colloidal particles can be utilized to form these hetero-aggregates, such as protein nanoparticles, nanoemulsion droplets, chitosan nanocrystals, or hydrogel particles. Consequently, it may be possible to build a wide range of materials with different microstructures and properties using this approach.

\subsection{Trojan horse nanoparticles}

Nanoparticles can be trapped within larger particles to enhance their functional attributes [82, 83]. For example, they may be trapped within hydrogel beads to improve their chemical and biochemical stability within food products, or to control their release characteristics within the human gastrointestinal tract (Figure 3). The concept of Trojanhorse nanoparticles is based on the Greek myth where the Greek army hid within a wooden horse so as to gain entry into the besieged city of Troy. Similarly, bioactiveloaded nanoparticles can be concealed within larger particles, and then released when they reach the site of action [82]. This concept has been used to encapsulate nanoemulsions in hydrogel beads so as to protect them in one environment, but then release them in another $[84,85]$. In these studies, nanoemulsions were prepared by microfluidization, and then mixed with an alginate solution. The resulting mixture was then injected into a calcium solution to form hydrogel beads with nanoscale oil droplets inside. The oil droplets could be released from these droplets by changing $\mathrm{pH}$ or ionic strength to alter the hydrogel pore size (Figure 3a) or to alter the attractive/repulsive interactions between the droplets and hydrogel matrix (Figure 3b). For example, the charge on protein-coated lipid nanoparticles goes from positive to negative when the $\mathrm{pH}$ is increased from below to above the isoelectric point. Consequently, the lipid droplets 
will be attracted to negatively charged alginate molecules at low $\mathrm{pH}$ and thus retained within the hydrogel beads, but repelled at high $\mathrm{pH}$ and thus released.

Trojan-horse nanoparticle systems may be used to protect oil droplets from lipid digestion within the upper gastrointestinal tract, and then release them within the colon [86]. They may also be used to control the digestion rate of lipid droplets within the small intestine, as well as the bioaccessibility of encapsulated hydrophobic bioactives (such as curcumin) [87]. In this case, curcumin-loaded nanoemulsion droplets were trapped inside polysaccharide beads formed using either alginate or carrageenan hydrogel beads. The gastrointestinal fate of the curcumin, determined using a simulated GIT, depended on the nature of the polysaccharide used to form the hydrogel beads. Recent studies have shown that hydrogel beads can also be used to encapsulate other types of nanoparticles, such as protein nanoparticles [88]. This may be useful for delivering bioactive peptides (or bioactives encapsulated within protein nanoparticles) to different regions of the GIT.

\subsection{Environmentally responsive nanoparticles}

For some applications it may be useful to have nanoparticles that change their properties in responses to specific environmental triggers, such as $\mathrm{pH}$, ionic strength, temperature, or enzyme activity. These food-grade nanoparticles may be useful in the development of diagnostic sensors or controlled release delivery systems. A number of recent studies have shown that nanoparticles can be developed that change properties in response to specific environmental triggers. In particular, there has been considerable research in the development of nanoparticles that dissociate and release their contents in response to specific enzymes. Bioactive-loaded lipid nanoparticles may be rapidly degraded and release their cargo when they are exposed to lipase under simulated GIT conditions, e.g., $\beta$-carotene [89, 90], fucoxanthin[91], curcumin [24, 92, 93], pterostilbene[94], Conenzyme Q10 [95], vitamin D [96], and vitamin E [97]. In this case, the bioactive components are primarily released in the small intestine due to the presence of pancreatic lipase. Similarly, bioactive-loaded protein nanoparticles may be rapidly degraded by proteases in the stomach or small intestine, e.g., riboflavin in whey protein nanoparticles [98], curcumin in zein nanoparticles [99], and resveratrol in zein nanoparticles [100]. In the pharmaceutical industry, $\mathrm{pH}$-sensitive nanoparticles have 
been developed to deliver anti-cancer components to tumors, e.g., by swelling or dissociating in response to a change in local $\mathrm{pH}$ [101]. Similar systems could be developed for utilization within the food industry to release active ingredients in response to a particular $\mathrm{pH}$ environment. For example, protein-chitosan nanoparticles have recently been developed that can release encapsulated hydrophobic bioactives in response to a pH trigger [102]. In this case, the biopolymer nanoparticles were assembled by electrostatic complexation of negative protein molecules with positive chitosan molecules.

\section{Conclusions}

In conclusion, it is difficult to accurately predict the future of food colloids as a scientific discipline since the driving forces for new research come from many different sources, and are constantly evolving. Nevertheless, it is safe to say that food colloids will continue to be important in the future. Colloidal particles play a critical role in most areas of the food industry, including food safety, quality, processing, packaging, and nutrition. Consequently, knowledge of their properties and behavior will always be essential for designing foods that are safer, higher quality, more nutritious, abundant, and sustainable. Having said this, there are some pressing societal needs where colloid science could have an important impact in the near future, including issues of sustainability, safety, and improved health.

Finally, it should be remarked that the future development of this field largely depends on the individuals who choose to work in it. Many researchers work on a particular topic because they were trained in that area by their academic advisors (including myself). However, the amount of resources devoted to these particular topics may not be proportionate to their scientific or societal importance. There may be many other topics that are more important or that require a different set of skills and approaches. Thus it is always important for researchers to continually evolve as new problems arise, and for the research field to welcome new researchers with different skills and experiences. 


\section{Acknowledgements}

This material was partly based upon work supported by the Cooperative State Research, Extension, Education Service, USDA, Massachusetts Agricultural Experiment Station (MAS00491) and USDA, NRI Grants (2013-03795).

\section{References}

1. Mackie A, Macierzanka A: Colloidal aspects of protein digestion.Current Opinion in Colloid \& Interface Science. 2010;15:102-8.

2. van Aken GA: Relating food emulsion structure and composition to the way it is processed in the gastrointestinal tract and physiological responses: What are the opportunities?Food Biophysics. 2010;5:258-83.

* A comprehensive review of the impact of food matrix properties (structure and composition) on the gastrointestinal fate of foods and their impact on human physiological responses (such as hunger and satiety). The article also describes how this knowledge can be used to develop novel functional foods to improve human health and wellness.

3. Kearney J: Food consumption trends and drivers.Philosophical Transactions of the Royal Society B-Biological Sciences. 2010;365:2793-807.

4. Vrieling A, Kampman E: The role of body mass index, physical activity, and diet in colorectal cancer recurrence and survival: a review of the literature.American Journal of Clinical Nutrition. 2010;92:471-90.

5. Pan H, Guo J, Su Z: Advances in understanding the interrelations between leptin resistance and obesity.Physiology \& Behavior. 2014;130:157-69.

6. Fardet A, Boirie Y: Associations between food and beverage groups and major diet-related chronic diseases: an exhaustive review of pooled/meta-analyses and systematic reviews.Nutrition Reviews. 2014;72:741-62.

7. Gupta SC, Kim JH, Prasad S, Aggarwal BB: Regulation of survival, proliferation, invasion, angiogenesis, and metastasis of tumor cells through modulation of inflammatory pathways by nutraceuticals.Cancer and Metastasis Reviews. 2010;29:405-34.

8. Laparra JM, Sanz Y: Interactions of gut microbiota with functional food components and nutraceuticals.Pharmacological Research. 2010;61:219-25. 9. Rubio L, Motilva M-J, Romero M-P: Recent advances in biologically active compounds in herbs and spices: A review of the most effective antioxidant and antiinflammatory active principles.Critical Reviews in Food Science and Nutrition. 2013;53:943-53.

10. McClements DJ, Xiao H: Potential biological fate of ingested nanoemulsions: influence of particle characteristics.Food \& Function. 2012;3:202-20.

11. Garrec DA, Frasch-Melnik S, Henry JVL, Spyropoulos F, Norton IT: Designing colloidal structures for micro and macro nutrient content and release in foods.Faraday Discussions. 2012;158:37-49. 
* A review of how foods can be designed to control the digestion of macronutrients (such as lipids, proteins, and starch) and the absorption of micronutrients (such as vitamins and nutraceuticals).

12. McClements DJ, Li F, Xiao H: The nutraceutical bioavailability classification scheme: Classifying nutraceuticals according to factors limiting their oral bioavailability. In: Doyle MP, Klaenhammer TR, editors. Annual Review of Food Science and Technology, Vol 62015. p. 299-327.

** This manuscript introduces a new classification scheme (NuBACS) that can be used to characterize the major factors limiting the bioavailability of nutraceuticals. Many of these factors require an understanding of the colloidal basis of food digestion.

13. McClements DJ: Reduced-Fat Foods: The Complex Science of Developing DietBased Strategies for Tackling Overweight and Obesity.Advances in Nutrition. 2015;6:338S-52S.

14. Floros JD, Newsome R, Fisher W, Barbosa-Canovas GV, Chen H, Dunne CP, et al.: Feeding the World Today and Tomorrow: The Importance of Food Science and Technology An IFT Scientific Review.Comprehensive Reviews in Food Science and Food Safety. 2010;9:572-99.

15. Weaver CM, Dwyer J, Fulgoni VL, King JC, Leveille GA, MacDonald RS, et al.: Processed foods: contributions to nutrition(1,2).American Journal of Clinical Nutrition. 2014;99:1525-42.

16. Lin CSK, Pfaltzgraff LA, Herrero-Davila L, Mubofu EB, Abderrahim S, Clark JH, et al.: Food waste as a valuable resource for the production of chemicals, materials and fuels. Current situation and global perspective.Energy \& Environmental Science. 2013;6:426-64.

17. Pfaltzgraff LA, De Bruyn M, Cooper EC, Budarin V, Clark JH: Food waste biomass: a resource for high-value chemicals.Green Chemistry. 2013;15:307-14. 18. Bacchin P, Marty A, Duru P, Meireles M, Aimar P: Colloidal surface interactions and membrane fouling: Investigations at pore scale.Advances in Colloid and Interface Science. 2011;164:2-11.

19. Du Z, Wang C, Tai X, Wang G, Liu X: Optimization and characterization of biocompatible oil-in-water nanoemulsion for pesticide delivery.Acs Sustainable Chemistry \& Engineering. 2016;4:983-91.

20. Oliveira AEMFM, Duarte JL, Amado JRR, Cruz RAS, Rocha CF, Souto RNP, et al.: Development of a larvicidal nanoemulsion with pterodon emarginatus vogel oil.Plos One. 2016;11.

21. Donsi F, Annunziata M, Sessa M, Ferrari G: Nanoencapsulation of essential oils to enhance their antimicrobial activity in foods.Lwt-Food Science and Technology. 2011;44:1908-14.

22. Donsi F, Annunziata M, Vincensi M, Ferrari G: Design of nanoemulsion-based delivery systems of natural antimicrobials: Effect of the emulsifier.Journal of Biotechnology. 2012;159:342-50.

23. Ziani K, Chang Y, McLandsborough L, McClements DJ: Influence of surfactant charge on antimicrobial efficacy of surfactant-stabilized thyme oil nanoemulsions.Journal of Agricultural and Food Chemistry. 2011;59:6247-55. 
24. Joung HJ, Choi M-J, Kim JT, Park SH, Park HJ, Shin GH: Development of foodgrade curcumin nanoemulsion and its potential application to food beverage system: Antioxidant property and in vitro digestion.Journal of Food Science. 2016;81:N745N53.

25. Maldonado-Valderrama J, Wilde P, Macierzanka A, Mackie A: The role of bile salts in digestion.Advances in Colloid and Interface Science. 2011;165:36-46.

26. Singh H: Aspects of milk-protein-stabilised emulsions.Food Hydrocolloids. 2011;25:1938-44.

27. Yao M, Xiao H, McClements DJ: Delivery of lipophilic bioactives: Assembly, disassembly, and reassembly of lipid nanoparticles. In: Doyle MP, Klaenhammer TR, editors. Annual Review of Food Science and Technology, Vol 52014. p. 53-81.

** This manuscript highlights the importance of designing the nanostructure and composition of food colloids so as to generate specific nanostructures in the small intestine (mixed micelles) and within the human body (chylomicrons) that may increase the bioavailability and bioactivity of nutraceuticals.

28. Pan K, Zhong Q: Organic nanoparticles in foods: Fabrication, characterization, and utilization. In: Doyle MP, Klaenhammer TR, editors. Annual Review of Food Science and Technology, Vol 72016. p. 245-66.

29. Froehlich E, Roblegg E: Models for oral uptake of nanoparticles in consumer products.Toxicology. 2012;291:10-7.

30. Oehlke K, Adamiuk M, Behsnilian D, Graef V, Mayer-Miebach E, Walz E, et al.:

Potential bioavailability enhancement of bioactive compounds using food-grade engineered nanomaterials: a review of the existing evidence.Food \& Function. 2014;5:1341-59.

** This article provides a comprehensive review of different approaches for increasing the bioavailability of nutraceuticals using colloidal delivery systems.

31. Fujimura KE, Slusher NA, Cabana MD, Lynch SV: Role of the gut microbiota in defining human health.Expert Review of Anti-Infective Therapy. 2010;8:435-54.

32. Jeffery IB, O'Toole PW: Diet-microbiota interactions and their implications for healthy living.Nutrients. 2013;5:234-52.

33. Baldwin EA, Bai J, Plotto A, Dea S: Electronic noses and tongues: Applications

for the food and pharmaceutical industries.Sensors. 2011;11:4744-66.

34. Kirsch J, Siltanen C, Zhou Q, Revzin A, Simonian A: Biosensor technology: recent advances in threat agent detection and medicine.Chemical Society Reviews. 2013;42:8733-68.

35. Van Dorst B, Mehta J, Bekaert K, Rouah-Martin E, De Coen W, Dubruel P, et al.: Recent advances in recognition elements of food and environmental biosensors: $A$ review.Biosensors \& Bioelectronics. 2010;26:1178-94.

36. Street RA, Ng TN, Schwartz DE, Whiting GL, Lu JP, Bringans RD, et al.: From printed transistors to printed smart systems.Proceedings of the Ieee. 2015;103:60718.

37. Liana DD, Raguse B, Gooding JJ, Chow E: Recent Advances in Paper-Based Sensors.Sensors. 2012;12:11505-26. 
38. Martin A, Vilela D, Escarpa A: Food analysis on microchip electrophoresis: An updated review.Electrophoresis. 2012;33:2212-27.

39. Yoon J-Y, Kim B: Lab-on-a-chip pathogen sensors for food safety.Sensors. 2012;12:10713-41.

40. Alting AC, van de Velde F: Proteins as clean label ingredients in foods and beverages. In: Baines D, Seal R, editors. Natural Food Additives, Ingredients and Flavourings2012. p. 197-211.

41. Ozturk B, Argin S, Ozilgen M, McClements DJ: Formation and stabilization of nanoemulsion-based vitamin $\mathrm{E}$ delivery systems using natural surfactants: Quillaja saponin and lecithin.Journal of Food Engineering. 2014;142:57-63.

42. Ozturk B, Argin S, Ozilgen M, McClements DJ: Formation and stabilization of nanoemulsion-based vitamin $\mathrm{E}$ delivery systems using natural biopolymers: Whey protein isolate and gum arabic.Food Chemistry. 2015;188:256-63.

43. Komaiko J, Sastrosubroto A, McClements DJ: Formation of oil-in-water emulsions from natural emulsifiers using spontaneous emulsification: Sunflower phospholipids.Journal of Agricultural and Food Chemistry. 2015;63:10078-88. 44. Karaca AC, Low NH, Nickerson MT: Potential use of plant proteins in the microencapsulation of lipophilic materials in foods.Trends in Food Science \& Technology. 2015;42:5-12.

* This article provides a nice overview of the utilization of plant proteins to create colloidal delivery systems for utilization in "all-natural" clean-label foods.

45. Lam RSH, Nickerson MT: Food proteins: A review on their emulsifying properties using a structure-function approach.Food Chemistry. 2013;141:975-84. * A nice review of the physiochemical basis for the utilization of food proteins to form and stabilize emulsions

46. Fisk ID, Linforth RST, Taylor AJ, Gray DA: Aroma encapsulation and aroma delivery by oil body suspensions derived from sunflower seeds (Helianthus annus).European Food Research and Technology. 2011;232:905-10.

47. Gallier S, Gordon KC, Singh H: Chemical and structural characterisation of almond oil bodies and bovine milk fat globules.Food Chemistry. 2012;132:1996-2006. 48. Adams GG, Imran S, Wang S, Mohammad A, Kok MS, Gray DA, et al.: Extraction, isolation and characterisation of oil bodies from pumpkin seeds for therapeutic use.Food Chemistry. 2012;134:1919-25.

49. Grundy MML, Carriere F, Mackie AR, Gray DA, Butterworth PJ, Ellis PR: The role of plant cell wall encapsulation and porosity in regulating lipolysis during the digestion of almond seeds.Food \& Function. 2016;7:69-78.

* A nice study highlighting the important role that the plant cell wall plays on the digestion of lipids in whole foods (such as nuts). This kind of information can be useful for developing novel delivery systems using biomimetic approaches.

50. Payne G, Lad M, Foster T, Khosla A, Gray D: Composition and properties of the surface of oil bodies recovered from Echium plantagineum. Colloids and Surfaces BBiointerfaces. 2014;116:88-92.

51. Mohit E, Rafati S: Biological delivery approaches for gene therapy: Strategies to potentiate efficacy and enhance specificity.Molecular Immunology. 2013;56:599-611. 
52. Su D, Zhong Q: Formation of thymol nanoemulsions with combinations of casein hydrolysates and sucrose stearate.Journal of Food Engineering. 2016;179:1-10.

53. Topuz OK, Ozvural EB, Zhao Q, Huang Q, Chikindas M, Golukcu M: Physical and antimicrobial properties of anise oil loaded nanoemulsions on the survival of foodborne pathogens.Food Chemistry. 2016;203:117-23.

54. Bhargava K, Conti DS, da Rocha SRP, Zhang Y: Application of an oregano oil nanoemulsion to the control of foodborne bacteria on fresh lettuce.Food Microbiology. 2015;47:69-73.

55. Landry KS, Micheli S, McClements DJ, McLandsborough L: Effectiveness of a spontaneous carvacrol nanoemulsion against Salmonella enterica Enteritidis and Escherichia coli 0157:H7 on contaminated broccoli and radish seeds.Food Microbiology. 2015;51:10-7.

56. McClements DJ: Food Emulsions: Principles, Practices, and Techniques. Third Edition ed. Boca Raton, FL: CRC Press; 2015.

57. Qian C, Decker EA, Xiao H, McClements DJ: Inhibition of beta-carotene degradation in oil-in-water nanoemulsions: Influence of oil-soluble and watersoluble antioxidants.Food Chemistry. 2012;135:1036-43.

58. Qian C, Decker EA, Xiao H, McClements DJ: Physical and chemical stability of beta-carotene-enriched nanoemulsions: Influence of $\mathbf{p H}$, ionic strength, temperature, and emulsifier type.Food Chemistry. 2012;132:1221-9.

59. Gowen AA, Feng YZ, Gaston E, Valdramidis V: Recent applications of hyperspectral imaging in microbiology.Talanta. 2015;137:43-54.

60. Schie IW, Huser T: Label-free analysis of cellular biochemistry by raman spectroscopy and microscopy.Comprehensive Physiology. 2013;3:941-56.

61. Wyroba E, Suski S, Miller K, Bartosiewicz R: Biomedical and agricultural applications of energy dispersive $\mathbf{X}$-ray spectroscopy in electron microscopy.Cellular \& Molecular Biology Letters. 2015;20:488-509.

62. Strohm EM, Moore MJ, Kolios MC: Single Cell Photoacoustic Microscopy: A

Review.Ieee Journal of Selected Topics in Quantum Electronics. 2016;22.

63. Yang ZB, Wang HB, Peng XY, Shi CC, Xia LP, Tang MJ, et al.: Recent progress in scanning probe microscope based super-resolution near-field fingerprint microscopy.Journal of Infrared and Millimeter Waves. 2016;35:87-98.

64. Ettelaie R: Computer simulation and modeling of food colloids.Current Opinion in Colloid \& Interface Science. 2003;8:415-21.

65. McClements DJ: Theoretical prediction of emulsion color.Advances in Colloid and Interface Science. 2002;97:63-89.

66. Pal R: Rheology of simple and multiple emulsions.Current Opinion in Colloid \& Interface Science. 2011;16:41-60.

67. Anzalone GC, Wijnen B, Pearce JM: Multi-material additive and subtractive prosumer digital fabrication with a free and open-source convertible delta RepRap 3-D printer.Rapid Prototyping Journal. 2015;21:506-19.

68. Furukawa H, Kawakami M, Gong J, Makino M, Kabir MH, Saito A: 3-D Gel

Printing for Soft-matter Systems Innovation. In: Varadan VK, editor. Nanosensors, Biosensors, and Info-Tech Sensors and Systems 20152015.

69. Huang QR, Yu HL, Ru QM: Bioavailability and delivery of nutraceuticals using nanotechnology.Journal of Food Science. 2010;75:R50-R7. 
70. McClements DJ: Emulsion Design to Improve the Delivery of Functional Lipophilic Components. In: Doyle MP, Klaenhammer TR, editors. Annual Review of Food Science and Technology, Vol 12010. p. 241-69.

71. Sagalowicz L, Leser ME: Delivery systems for liquid food products.Current Opinion in Colloid \& Interface Science. 2010;15:61-72.

72. McClements DJ: Advances in fabrication of emulsions with enhanced functionality using structural design principles.Current Opinion in Colloid \& Interface Science. 2012;17:235-45.

73. Patel AR, Velikov KP: Colloidal delivery systems in foods: A general comparison with oral drug delivery.LWT-Food Science and Technology. 2011;44:1958-64.

* This article highlights the similarities between the approaches taken in the food and pharmaceutical industries for developing delivery systems for oral delivery of bioactive agents.

74. McClements DJ: Nanoparticle- and Microparticle-based Delivery Systems. Boca Raton, FL: CRC Press; 2014.

* A recent book that provides a comprehensive overview of the many different kinds of colloidal delivery systems available for delivery of bioactive agents, including lipidbased, biopolymer-based, and surfactant-based systems.

75. Livney YD: Nanostructured delivery systems in food: latest developments and potential future directions.Current Opinion in Food Science. 2015;3:125-35.

** This article provides a review of nanostructured approaches for increasing developing food-grade colloidal delivery systems with enhanced performance.

76. Fathi M, Mozafari MR, Mohebbi M: Nanoencapsulation of food ingredients using lipid based delivery systems.Trends in Food Science \& Technology. 2012;23:13-27.

77. Zou L, Zheng B, Zhang R, Zhang Z, Liu W, Liu C, et al.: Enhancing the bioaccessibility of hydrophobic bioactive agents using mixed colloidal dispersions: Curcumin-loaded zein nanoparticles plus digestible lipid nanoparticles.Food Research International. 2016;81:74-82.

78. Chen J, Zheng J, Decker EA, McClements DJ, Xiao H: Improving nutraceutical bioavailability using mixed colloidal delivery systems: lipid nanoparticles increase tangeretin bioaccessibility and absorption from tangeretin-loaded zein nanoparticles.RSC Advances. 2015;5:73892-900.

* A recent research paper that highlights the potential of using mixed colloidal delivery systems to improve their efficacy. For example, one colloidal particle (zein nanoparticles) can be used to achieve a high bioactive loading, wereas another colloidal particle (lipid droplets) can be used to increase bioaccessibility.

79. Mao Y, McClements DJ: Modulation of food texture using controlled heteroaggregation of lipid droplets: Principles and applications.Journal of Applied Polymer Science. 2013;130:3833-41.

80. Maier C, Zeeb B, Weiss J: Investigations into aggregate formation with oppositely charged oil-in-water emulsions at different $\mathbf{p H}$ values.Colloids and Surfaces B-Biointerfaces. 2014;117:368-75. 
81. Mao Y, McClements DJ: Modification of emulsion properties by heteroaggregation of oppositely charged starch-coated and protein-coated fat droplets.Food Hydrocolloids. 2013;33:320-6.

82. Zhang Z, Zhang R, Chen L, Tong Q, McClements DJ: Designing hydrogel particles for controlled or targeted release of lipophilic bioactive agents in the gastrointestinal tract.European Polymer Journal. 2015;72:698-716.

83. van Leusden P, den Hartog GJM, Bast A, Postema M, van der Linden E, Sagis LMC: Strength of microbeads for the encapsulation of heat sensitive, hydrophobic components.Food Hydrocolloids. 2016;56:318-24.

84. Zeeb B, Saberi AH, Weiss J, McClements DJ: Formation and characterization of filled hydrogel beads based on calcium alginate: Factors influencing nanoemulsion retention and release.Food Hydrocolloids. 2015;50:27-36.

85. Zeeb B, Saberi AH, Weiss J, McClements DJ: Retention and release of oil-inwater emulsions from filled hydrogel beads composed of calcium alginate: impact of emulsifier type and pH.Soft Matter. 2015;11:2228-36.

86. Li Y, Hu M, Du YM, Xiao H, McClements DJ: Control of lipase digestibility of emulsified lipids by encapsulation within calcium alginate beads.Food Hydrocolloids. 2011;25:122-30.

87. Zhang ZP, Zhang RJ, Zou LQ, Chen L, Ahmed Y, Al Bishri W, et al.:

Encapsulation of curcumin in polysaccharide-based hydrogel beads: Impact of bead type on lipid digestion and curcumin bioaccessibility.Food Hydrocolloids.

2016;58:160-70.

88. Zou LQ, Zhang ZP, Zhang RJ, Liu W, Liu CM, Xiao H, et al.: Encapsulation of protein nanoparticles within alginate microparticles: Impact of $\mathbf{p H}$ and ionic strength on functional performance.Journal of Food Engineering. 2016;178:81-9. 89. Zhang R, Zhang Z, Kumosani T, Khoja S, Abualnaja KO, McClements DJ:

Encapsulation of beta-carotene in Nanoemulsion-Based Delivery Systems Formed by Spontaneous Emulsification: Influence of Lipid Composition on Stability and Bioaccessibility.Food Biophysics. 2016;11:154-64.

90. Yi J, Zhong F, Zhang Y, Yokoyama W, Zhao L: Effects of lipids on in vitro release and cellular uptake of beta-carotene in nanoemulsion-based delivery systems.Journal of Agricultural and Food Chemistry. 2015;63:10831-7.

91. Salvia-Trujillo L, Sun Q, Urn BH, Park Y, McClements DJ: In vitro and in vivo study of fucoxanthin bioavailability from nanoemulsion-based delivery systems: Impact of lipid carrier type.Journal of Functional Foods. 2015;17:293-304.

92. Majeed H, Antoniou J, Hategekimana J, Sharif HR, Haider J, Liu F, et al.: Influence of carrier oil type, particle size on in vitro lipid digestion and eugenol release in emulsion and nanoemulsions.Food Hydrocolloids. 2016;52:415-22.

93. Pinheiro AC, Coimbra MA, Vicente AA: In vitro behaviour of curcumin nanoemulsions stabilized by biopolymer emulsifiers - Effect of interfacial composition.Food Hydrocolloids. 2016;52:460-7.

94. Sun Y, Xia Z, Zheng J, Qiu P, Zhang L, McClements DJ, et al.: Nanoemulsionbased delivery systems for nutraceuticals: Influence of carrier oil type on bioavailability of pterostilbene.Journal of Functional Foods. 2015;13:61-70. 
95. Cho HT, Salvia-Trujillo L, Kim J, Park Y, Xiao H, McClements DJ: Droplet size and composition of nutraceutical nanoemulsions influences bioavailability of long chain fatty acids and Coenzyme Q10.Food Chemistry. 2014;156:117-22.

96. Ozturk B, Argin S, Ozilgen M, McClements DJ: Nanoemulsion delivery systems for oil-soluble vitamins: Influence of carrier oil type on lipid digestion and vitamin D-3 bioaccessibility.Food Chemistry. 2015;187:499-506.

97. Mayer S, Weiss J, McClements DJ: Behavior of vitamin E acetate delivery systems under simulated gastrointestinal conditions: Lipid digestion and bioaccessibility of low-energy nanoemulsions.Journal of Colloid and Interface Science. 2013;404:215-22.

98. Madalena DA, Ramos OL, Pereira RN, Bourbon AI, Pinheiro AC, Malcata FX, et al.: In vitro digestion and stability assessment of beta-lactoglobulin/riboflavin nanostructures.Food Hydrocolloids. 2016;58:89-97.

99. Zou L, Zheng B, Zhang R, Zhang Z, Liu W, Liu C, et al.: Food-grade nanoparticles for encapsulation, protection and delivery of curcumin: comparison of lipid, protein, and phospholipid nanoparticles under simulated gastrointestinal conditions.Rsc Advances. 2016;6:3126-36.

* This study compares the advantages and limitations of different kinds of colloidal delivery systems.

100. Davidov-Pardo G, Perez-Ciordia S, Marin-Arroyo MR, McClements DJ: Improving resveratrol bioaccessibility using biopolymer nanoparticles and complexes: Impact of protein-carbohydrate maillard conjugation.Journal of Agricultural and Food Chemistry. 2015;63:3915-23. 101. Liu J, Huang YR, Kumar A, Tan A, Jin SB, Mozhi A, et al.: pH-sensitive nanosystems for drug delivery in cancer therapy.Biotechnology Advances. 2014;32:693710.

102. Kurukji D, Norton I, Spyropoulos F: Fabrication of sub-micron protein-chitosan electrostatic complexes for encapsulation and pH-Modulated delivery of model hydrophilic active compounds.Food Hydrocolloids. 2016;53:249-60. 


\section{Graphical Abstract}

\section{Bioactive-loaded Protein nanoparticles}

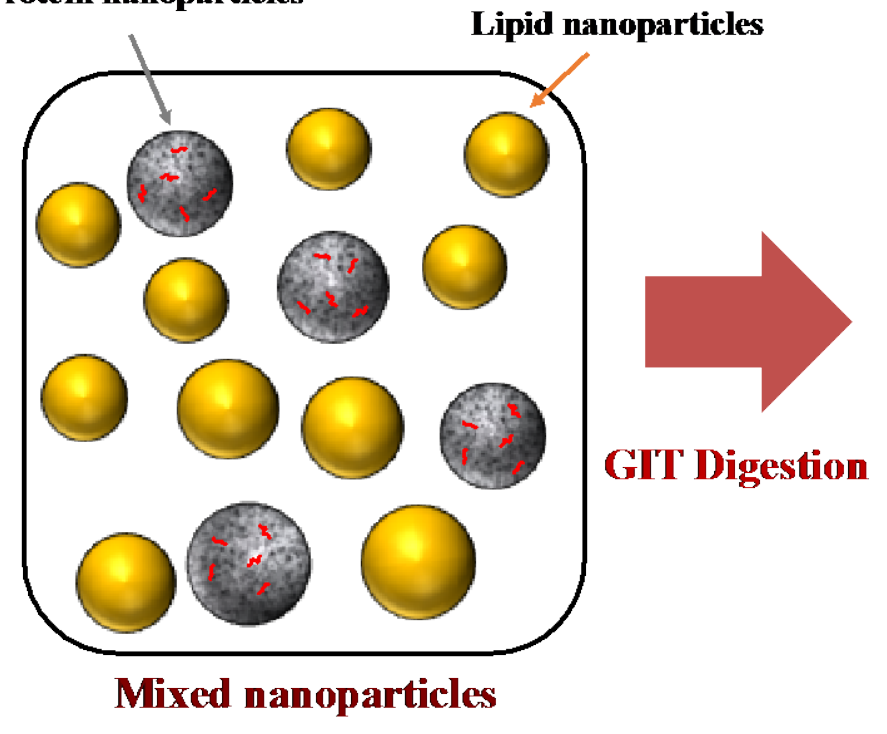

Digestible

ipid nanoparticles

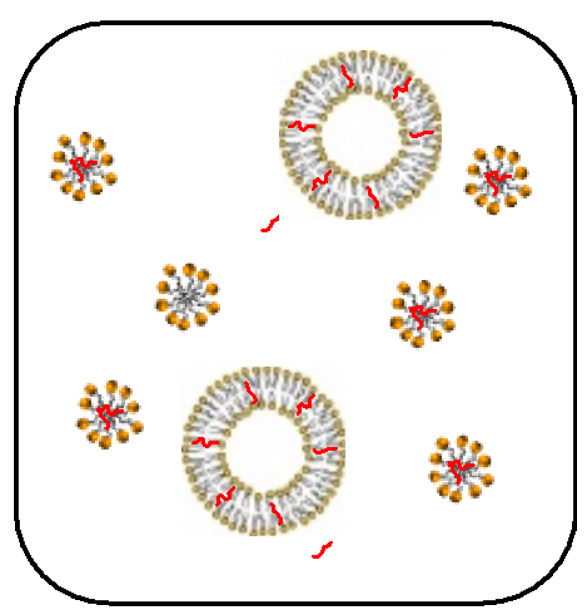

Micelles \& vesicles 
Figure 1: Mixed nanoparticle delivery systems can be designed to increase the oral bioavailability of hydrophobic bioactive agents. In this case, hydrophobic bioactives are loaded into protein-nanoparticles that are mixed with digestible lipid nanoparticles.

\section{Bioactive-loaded}

Protein nanoparticles

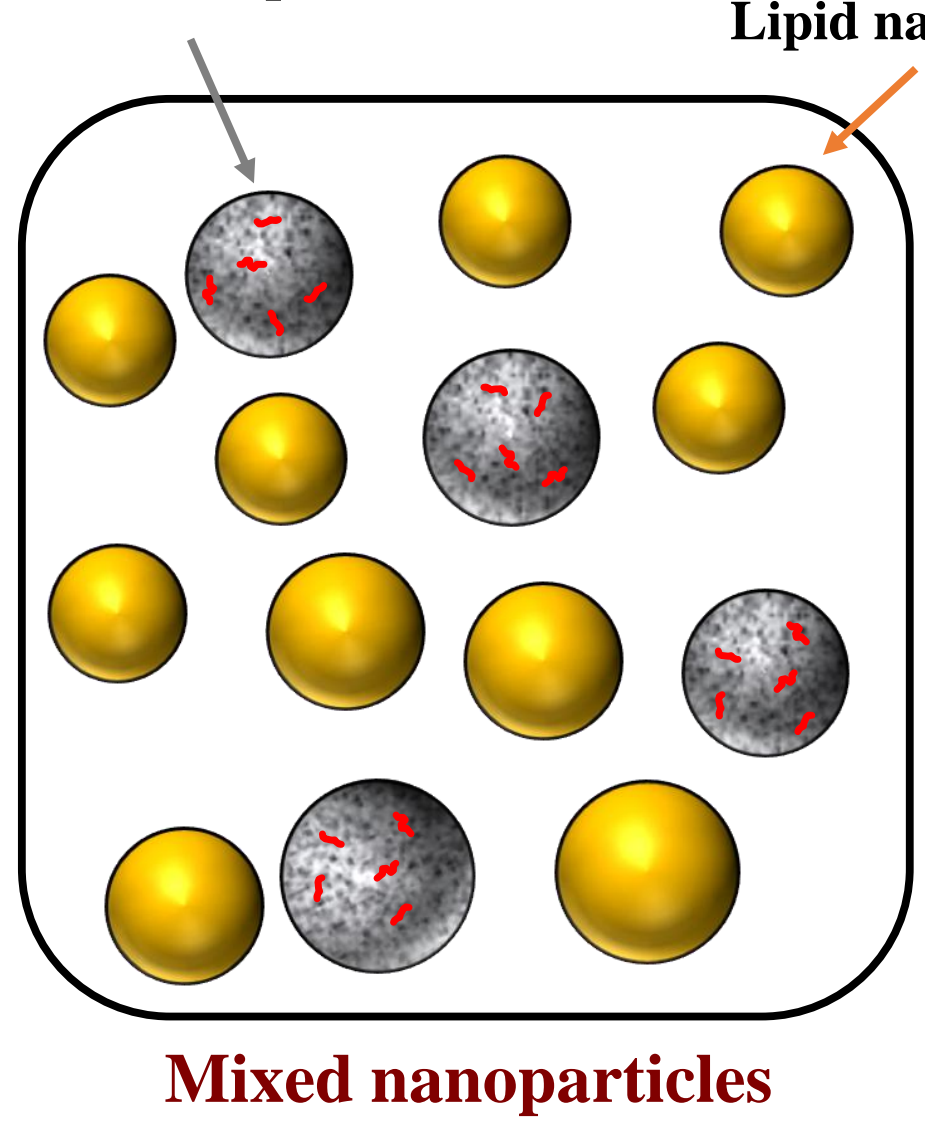

Digestible

Lipid nanoparticles
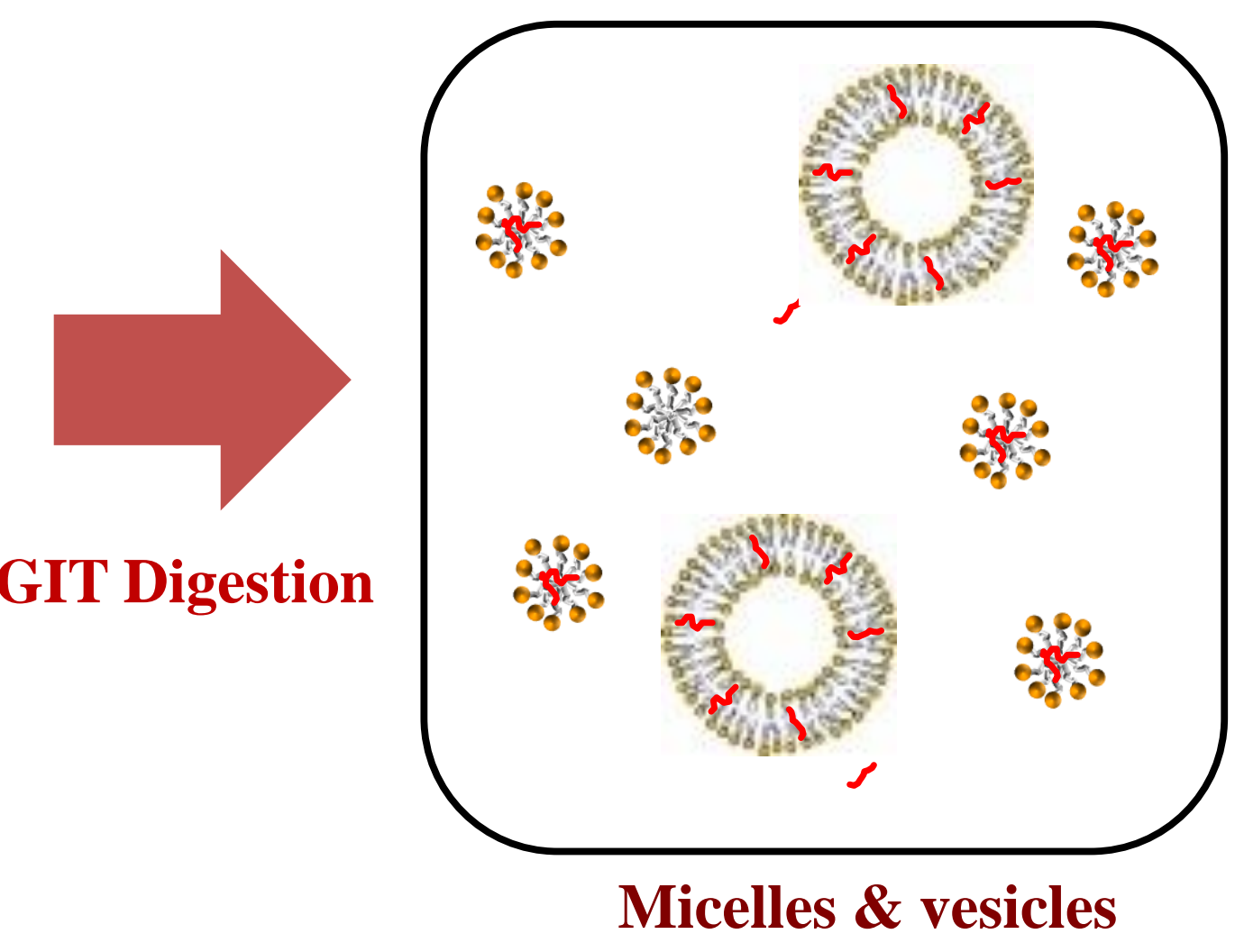
Figure 2: Colloidal dispersions with novel rheological properties can be prepared by mixing positive and negative nanoparticles together to induce heteroaggregation.
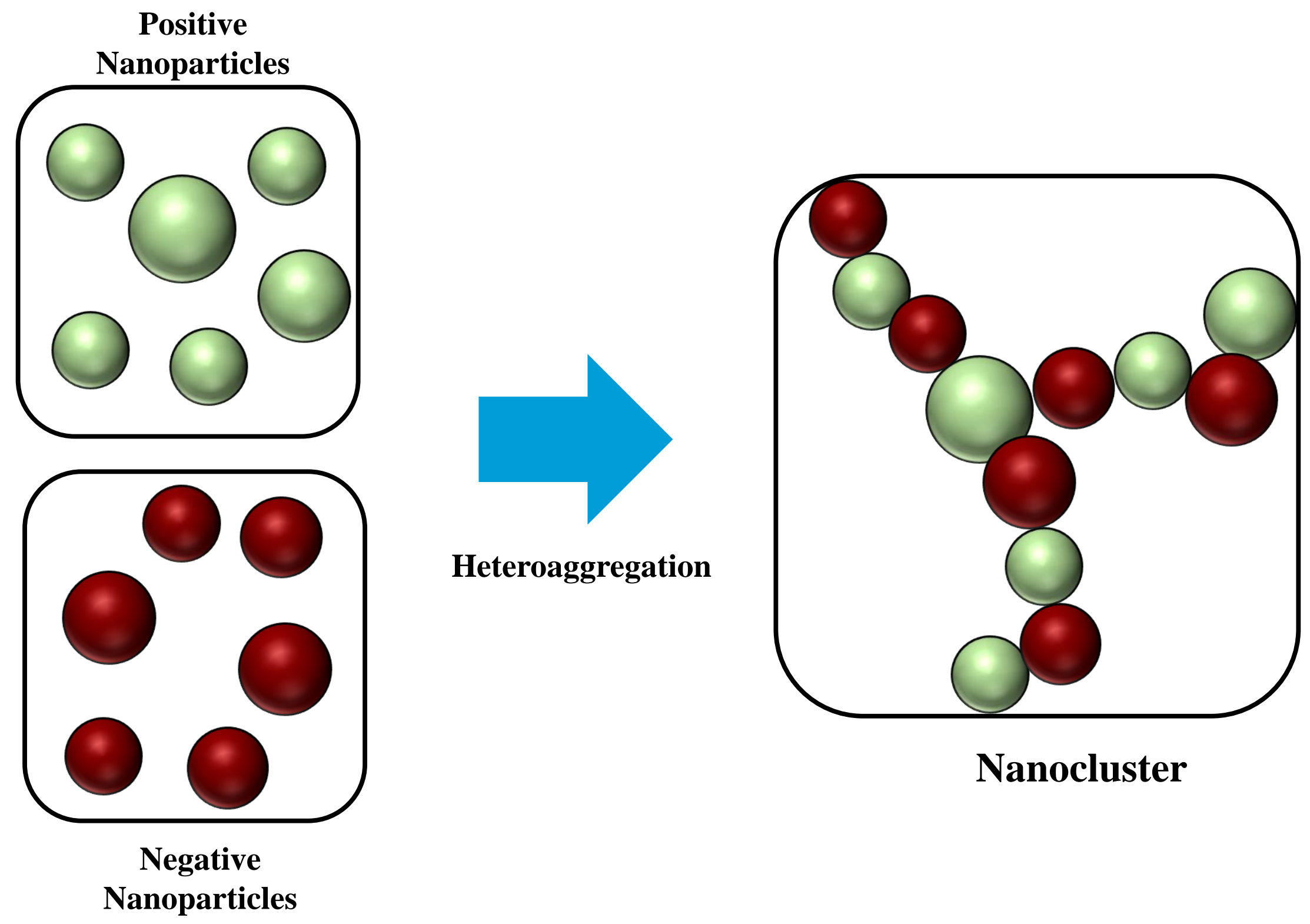

\section{Nanoparticles}


Figure 3a: Lipid nanoparticles may be trapped inside larger particles (such as hydrogel beads) and their release controlled by swelling due to an increase in pore size.

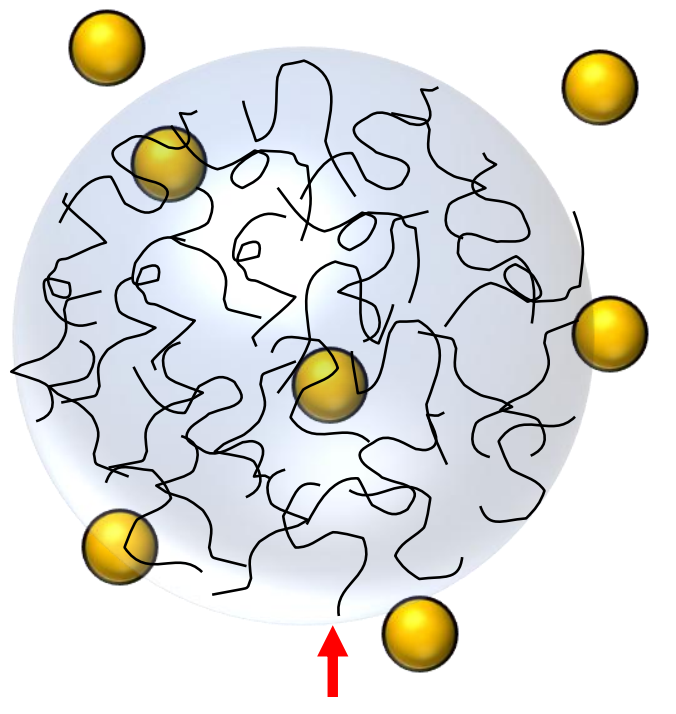

Release

Retention

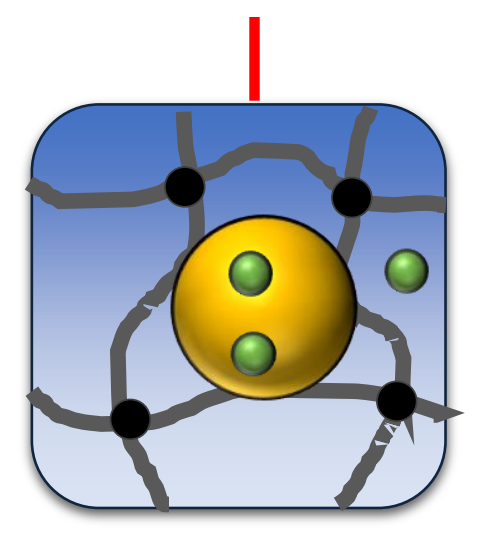

Small Pores
Trigger
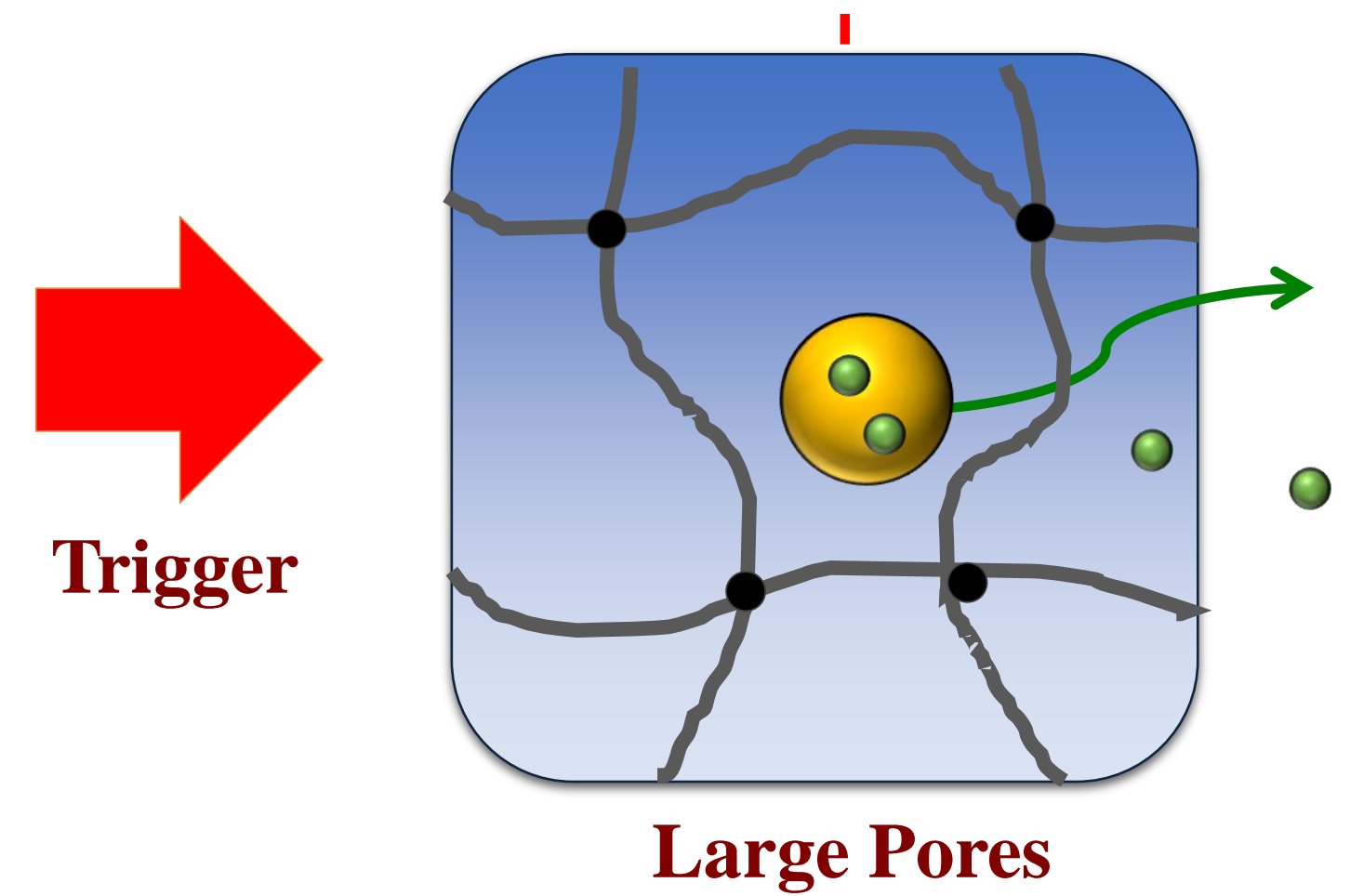
Figure 3b: Lipid nanoparticles may be trapped inside larger particles (such as hydrogel beads) and their release controlled by a change from attractive to repulsive interactions.
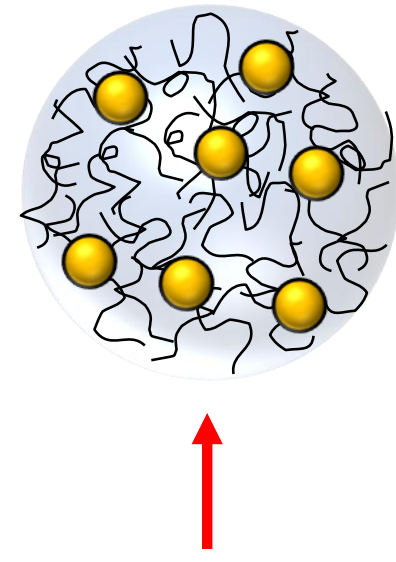

Retention

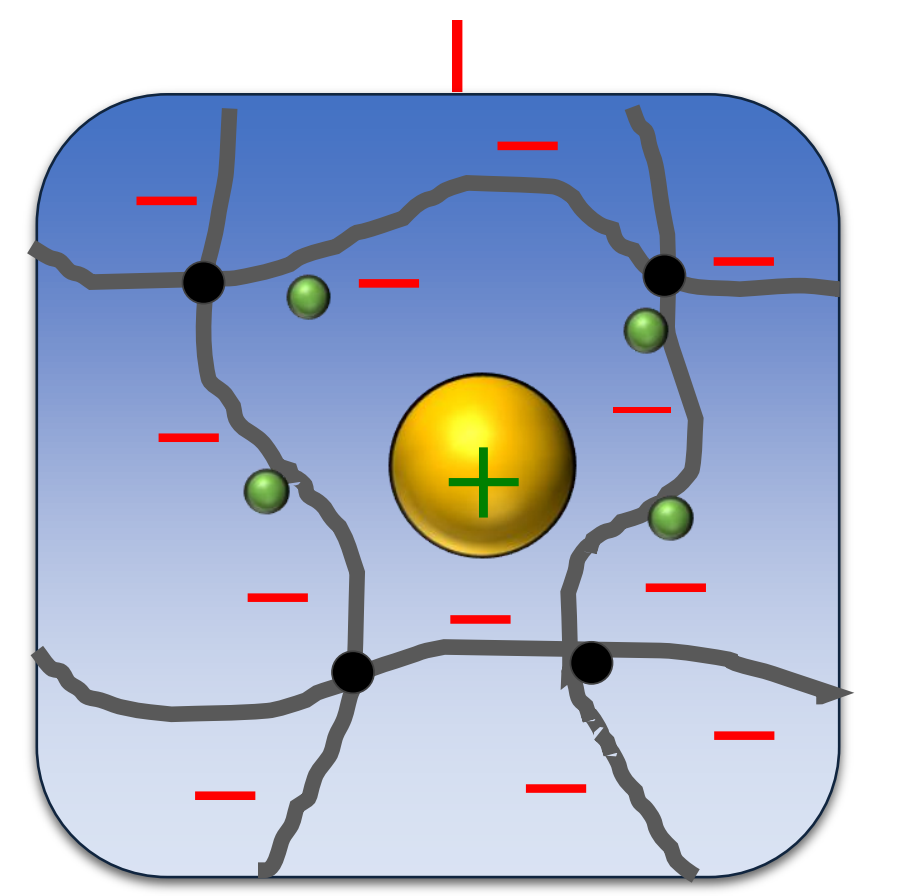

Attractive

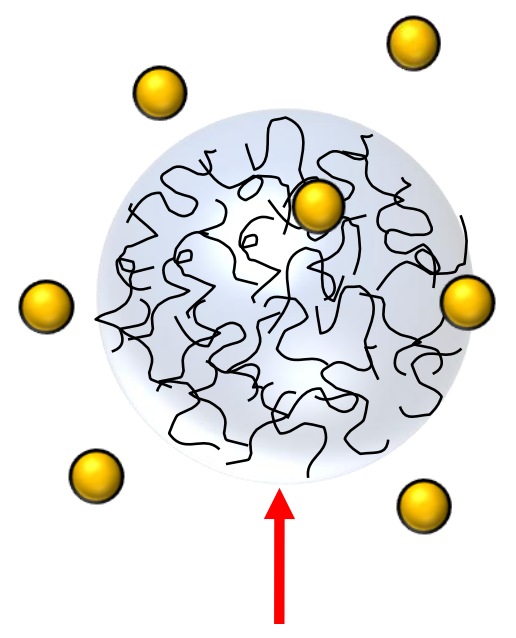

Release

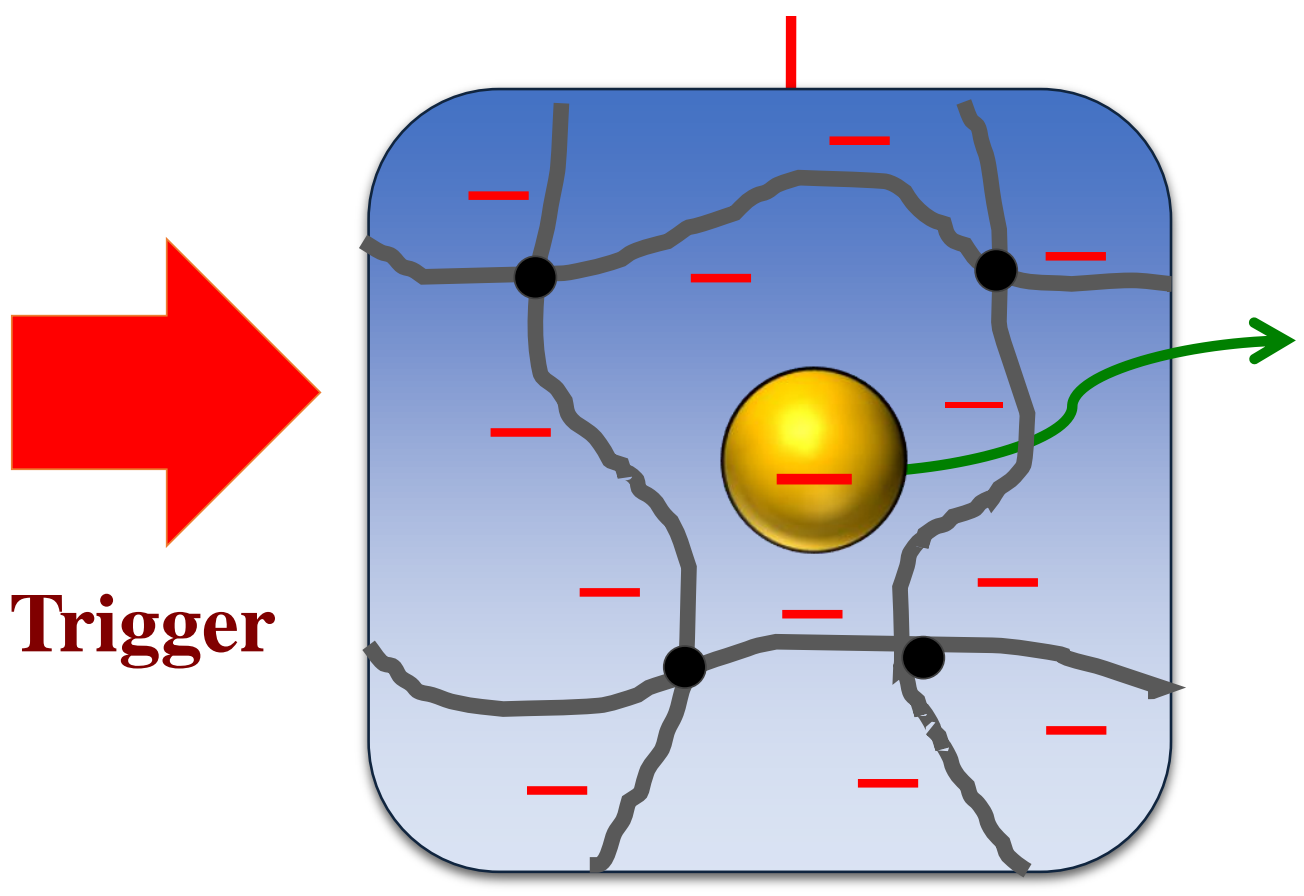

Repulsive 